\title{
INTEGRACIÓN DE PLANTACIONES FORESTALES COMERCIALES COLOMBIANAS EN CONCEPTOS DE BIORREFINERÍA TERMOQUÍMICA: UNA REVISIÓN
}

\section{Integration of Colombians Forest Commercial Crops in Thermochemical Biorefinery Concepts: A Review}

\author{
Juan F. Pérez ${ }^{1}$ Rolando Barrera² \& Gloria Ramírez ${ }^{3}$
}

Pérez, J., Barrera, R. \& Ramírez, G. (2015). Integración de plantaciones forestales comerciales colombianas en conceptos de biorrefinería termoquímica: una revisión. Colombia Forestal, 18(2),273-294.

Recepción: 25 de septiembre de 2014

\section{RESUMEN}

La integración de las plantaciones forestales comerciales colombianas en conceptos de biorrefinería se justifica desde las perspectivas técnica, energética, social y ambiental; adicionalmente se enmarca dentro de diversos planes y políticas de gobierno que consideran el aprovechamiento energético de la biomasa como alternativa recursiva para el potencial silvicultural del país. En este trabajo se revisan algunos procesos específicos que pueden evaluarse como posibles estrategias de integración de la madera plantada en Colombia bajo el concepto de biorrefinería. Se abarcan procesos de gasificación de baja-media potencia, gasificación a escala industrial para producción de biocombustibles de alta calidad, procesos de mejoramiento de la madera como biocombustible sólido, y la producción de biocarbón mediante métodos alternativos; por último, se revisa el mercado potencial de productos forestales de valor agregado. A modo de conclusión se destaca el alto potencial forestal de Colombia, por tanto, la unión estratégica entre universidades, centros de investigación y
Aprobación: 2 de marzo de 2015

el sector forestal debe buscar la eficiencia e innovar para ofrecer productos diferenciadores y con valor agregado, aprovechando la existencia de nichos de mercado prácticamente inexplorados en Colombia, como la bioenergía y los bioproductos. A ese punto se dirige esta revisión, pues es necesario avanzar en el conocimiento que se tiene de las características y posibles usos de algunas especies forestales que se cultivan en el país desde conceptos de biorrefinería para bioenergía, biocombustibles y bioproductos bajo una perspectiva de sostenibilidad.

Palabras clave: bioenergía, biomasa, bioproductos, biorrefinería termoquímica, plantaciones forestales comerciales.

\footnotetext{
ABSTRACT

The technical, energy, social and environmental benefits of the integration of commercial forest crops in Colombia under biorefinery concepts are evaluated. This concept is part of various programs and government policies that consider the energy
}

Grupo de Manejo Eficiente de la Energía (Gimel), Departamento de Ingeniería Mecánica, Facultad de Ingeniería, Universidad de Antioquia, Calle 70 No.52-21, Medellín, Colombia. juanpb@udea.edu.co.

2 Grupo CERES, Departamento de ingeniería química, Facultad de Ingeniería, Universidad de Antioquia, Calle 70 No. 52-21, Medellín, Colombia. rolando.barrera@gmail.com.

3 Grupo GESTA, Departamento de ingeniería industrial, Facultad de Ingeniería, Universidad de Antioquia, Calle 70 No. 52-21, Medellín, Colombia.glorial.ramirez@udea.edu.co. Autor de correspondencia. 
use of biomass as an alternative source to the silvicultural potential of the country. In this paper we review some specific processes that can be evaluated as integration strategies with high potential to use the wood planted in Colombia under biorefinery concepts. The processes considered are low-middle power gasification, industrial scale gasification to high quality biofuel production, wood pretreatment to improve the solid biofuel and alternative methods for biochar production. Finally, we also review the value-added wood products market. To conclude we highlight the potential of Colombian forest in this industry, Through strategic alliances between universities, research centers and the forestry sector, more efficient and innovative development of new valueadded products should be sought, taking advantage of the unexplored market opportunities in Colombia for bioenergy and bioproducts. This review aims to advance knowledge on the features and possible uses of forest species to produce bioenergy, biofuels, and bioproducts sustainably.

Key words: bioenergy, wood biomass, bioproducts, thermochemical biorefinery, forest commercial crops.

\section{INTRODUCCIÓN}

La Organización de las Naciones Unidas para la Agricultura y la Alimentación (FAO), en su estudio titulado "Tendencias y perspectivas del sector forestal en América Latina y el Caribe", establece que los países con mayor desarrollo forestal-industrial de América Latina ( $A L)$ y el Caribe en producción, consumo y comercio, principalmente de pasta y papel, madera aserrada, tableros de madera, además de leña (biomasa) y carbón vegetal, son Brasil, Chile, México, Colombia, Venezuela, Argentina, Uruguay y Ecuador. Por otro lado, los cinco países con mayor cobertura forestal son Brasil (477.7 millones de hectáreas (ha)), Perú (68.7 millones ha), México (64.2 millones ha), Colombia (60.7 millones ha) y Bolivia (58.7 millones ha), que constituyen el $79 \%$ del área forestal total en $A L$ y el Caribe. Con respecto a los bosques plantados, Brasil, Chile, Argentina y Uruguay poseen el $88 \%$ del total de la superficie plantada en la región.

Según el estudio, se visualiza un crecimiento en la producción industrial del sector forestal, mas no por aumento en el consumo sino por mayores exportaciones de madera o derivados. Los principales productos exportados son pasta y papel, madera aserrada, contrachapado y algunos productos de mayor valor agregado como molduras de pino, pisos y muebles. De los productos forestales para energía, en la región se destaca el consumo de biomasa para cocción principalmente en Centro América y el Caribe; mientras que en el Cono Sur, Brasil produce el $80 \%$ del carbón vegetal de AL para el autoconsumo en la industria siderúrgica. No obstante, se destaca que la biomasa como fuente de energía es un tema poco conocido en la mayoría de los países de la región pese a su importancia económica (FAO, 2006).

Colombia viene adelantando un marco legal que busca fortalecer el sector forestal. En el año 2000, el Plan Nacional de Desarrollo Forestal definió la base estratégica hacia el año 2025 con principios de sostenibilidad de los bosques naturales y plantados (Minambiente, 2000). En el año 2011, el Acuerdo de Competitividad Forestal Nacional establece como objetivo para el año 2025 consolidar la cadena productiva forestal (maderas, tableros, muebles y productos de madera) como vector estratégico del desarrollo económico nacional, mediante la participación en el mercado local y global (Fedemaderas, 2011; Minambiente, 2000).

Con respecto al potencial forestal, Colombia cuenta con 17 millones de hectáreas aptas para reforestación, de las cuales el 30\% no tiene restricciones. Para el año 2011 solo se tenían 364080 ha con plantaciones forestales comerciales, lo cual representa una gran oportunidad de crecimiento y desarrollo para el país en el marco del Plan 
Nacional de Desarrollo 2010-2014 (PND) (Fedemaderas, 2011; Minambiente et al., 2011), cuyo programa de reforestación plantea alcanzar 1382 064 ha reforestadas para el año 2019. Este vector estratégico del sector agropecuario también busca contribuir al desarrollo económico y social del país, con una tasa de crecimiento del 5.3\%, y con generación de empleo, que a mediano plazo puede ser soporte de cara a un eventual proceso de paz (CNPES, 2013; DNP, 2010). No obstante, atendiendo conceptos de competitividad y globalización, es necesario buscar usos alternativos y sostenibles de las maderas comerciales como la bioenergía y los bioproductos, que generen mayor valor agregado a los recursos forestales. Este nuevo aspecto, poco explorado en el país, deja al descubierto la necesidad de estudiar la biorrefinería forestal como alternativa energética y fuente de productos de alto valor agregado para el sector.

Integrar las plantaciones forestales comerciales en conceptos de biorrefinería contribuye a mitigar la problemática energética, ambiental y social causada por la energía de origen fósil. Se destaca que a nivel global en la última década se ha mejorado el rendimiento de los sistemas energéticos; sin embargo, el consumo de combustibles fósiles sigue aumentando (Figura 1). Según la Agencia de
Administración de Información de la Energía de los Estados Unidos, en el 2011 el consumo energético global alcanzó los 531.2 cuatrillones de BTU (British Thermal Unit), con un crecimiento anual aproximado del $1.6 \%$; crecimiento que se proyecta hasta el año 2035 debido al continuo aumento de equipos, procesos y máquinas que demandan energía (EIA, 2011). Una alternativa que promete reducir la dependencia de energéticos de origen fósil es la investigación y desarrollo de las fuentes de energía renovables. La biomasa contribuye con cerca del $14 \%$ del suministro de energía primaria global, y es considerada la cuarta fuente energética (Lighong et al., 2010). Con el estudio e implementación de sistemas que operen con estas fuentes se puede diversificar la canasta energética, reducir la dependencia de los países que no disponen de suficiente energía primaria fósil para su autoabastecimiento y mitigar los impactos medioambientales ocasionados por el uso de combustibles de origen fósil, como el cambio climático (Ghatak, 2011; Lighong et al., 2010).

Además del sector energético, es necesario encontrar materias primas alternativas al petróleo para la industria química, ya que esta industria depende en un $90 \%$ de este recurso fósil. Entre varios materiales explorados, la biomasa es el único que tiene ese potencial de aplicación dual (Ghatak, 2011).

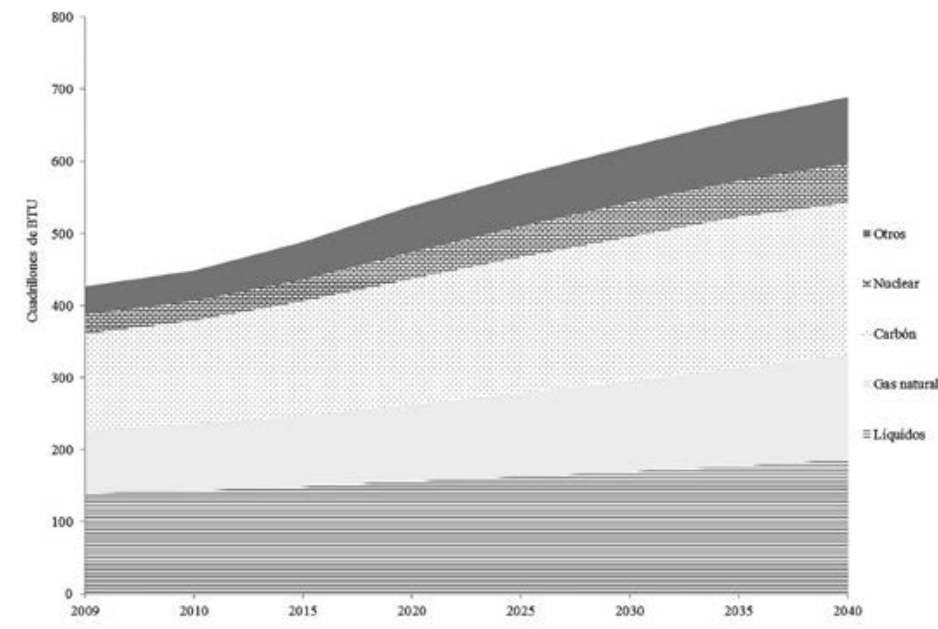

a)

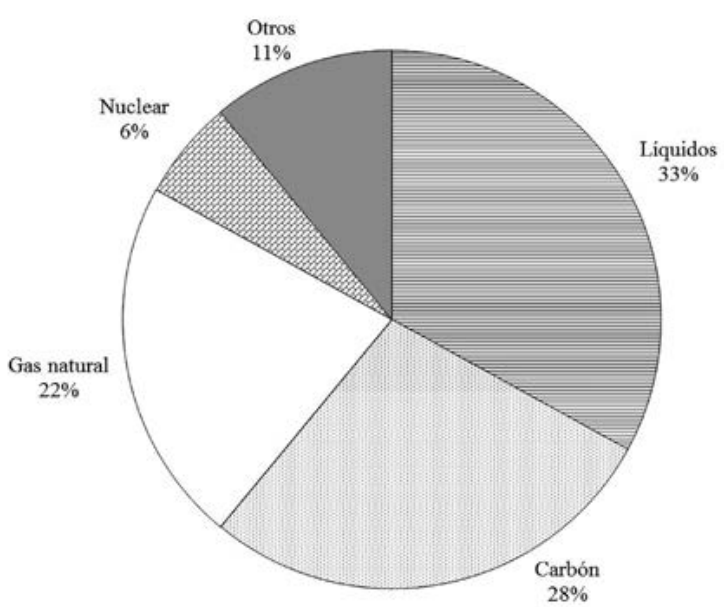

b)

Figura 1. Consumo total de energía a nivel global. a) Prospectiva de evolución del consumo b) Canasta energética a 2011 (EIA, 2010). 
Actualmente se dispone de varios sistemas de diferente madurez tecnológica que utilizan fuentes primarias renovables como la energía solar, eólica, hidráulica, biomásica, entre otras, que podrían ser utilizadas para la generación de potencia y solucionar varios de los inconvenientes asociados al uso de los combustibles fósiles, tales como su carácter agotable y emisiones contaminantes con impactos ambientales globales. Por esta razón, y teniendo en cuenta que la biomasa representa entre el 10 y el $15 \%$ de la energía primaria global ( $\mathrm{Li}$ et al., 2008), se viene promoviendo su uso para la generación de bioenergía y bioproductos en el marco de biorrefinería, buscando maximizar el rendimiento con el menor impacto ambiental ( $\mathrm{Vi}$ shnu \& Mala, 2012).

La integración de las plantaciones forestales comerciales colombianas en conceptos de biorrefinería se justifica desde las perspectivas técnica, energética, social y ambiental; adicionalmente se enmarca dentro de planes de gobierno como el Plan Energético Nacional 2006-2025, Plan de Desarrollo Para Fuentes No Convencionales de Energía, y el Programa de Uso Racional y Eficiente de Energía y Fuentes No Convencionales 2010-2015 (UPME, 2007; 2010c). Tales políticas consideran el aprovechamiento energético de biomasa como una alternativa de gran potencial para suplir las demandas energéticas de las zonas no interconectadas (ZNI) colombianas, y destacan los bajos costos de generación de estas tecnologías en comparación con los obtenidos en plantas diésel, las cuales suplen cerca del 90\% de la generación eléctrica en las ZNI (Minminas, 2010; UPME, 2010c). Las políticas gubernamentales buscan impulsar el desarrollo del sector forestal bajo la premisa de sostenibilidad y desarrollo nacional, e impactar las zonas rurales del país con desarrollo y calidad de vida, lo cual reducirá el éxodo a las grandes ciudades y brindará oportunidades de empleo y educación (Rincón-Narváez, 2010).

Por otro lado, el estudio e investigación en sistemas de aprovechamiento energético de la biomasa (biocombustibles) es una alternativa global viable para contrarrestar el carácter finito de los combustibles fósiles y la creciente contaminación asociada a su combustión (Nenoff et al., 2012). La biomasa contribuye al reciclaje de $\mathrm{CO}_{2}$ en la atmósfera sin contribuir al efecto invernadero, esto se debe a que el $\mathrm{CO}_{2}$ que libera la biomasa durante la combustión corresponde a la misma cantidad que consume de la atmósfera durante su crecimiento (Demirbas et al., 2009).

Un aspecto adicional que asegura la calidad y pertinencia de la integración de la madera forestal comercial de Colombia en conceptos de biorrefinería es el potencial silvicultural del país, pues los rendimientos del país para algunas de las principales especies forestales comerciales están entre $26-30 \mathrm{~m}^{3} / \mathrm{ha} /$ año para acacia, $15-40 \mathrm{~m}^{3} / \mathrm{ha} / \mathrm{año}$ para eucalipto, $20-25 \mathrm{~m}^{3} / \mathrm{ha} / \mathrm{año} \mathrm{para} \mathrm{melina} \mathrm{y} 30$ -

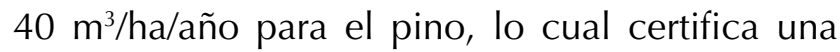
alta producción de madera, por lo que Colombia juega un papel importante en Latinoamérica ( $F A O$, 2006; Fedemaderas, 2011). Además, dichos cultivos dedicados a biorrefinerías no compiten con la agricultura y pueden crecer en suelos degradados (Osorio et al., 2014).

Esta línea estratégica del país debe ir acompañada del aumento de la demanda de productos maderables, dada la existencia de nichos de mercado prácticamente inexplorados en Colombia, como la bioenergía y los bioproductos. Los productos de una biorrefinería no son solamente biocombustibles sólidos (pélets, lignina y biocarbones), líquidos (bioetanol, biodiésel, combustibles Fischer-Tropsch y bioaceites) y gaseosos (biogás, syngás, hidrógeno y biometano), sino también materiales y productos químicos como ácidos, polímeros, resinas y fertilizantes que podrían resultar de utilidad en diversos sectores industriales (Cherubini, 2010; Octave \& Thomas, 2009). Por lo anterior, en este trabajo se revisan algunos procesos específicos que pueden evaluarse como posibles estrategias de integración de la madera plantada en Colombia bajo el concepto de biorrefinería. Se abarcan procesos de gasificación de baja-media potencia, gasificación a escala industrial para producción de 
biocombustibles de alta calidad $\left(\mathrm{H}_{2}\right.$ y $\left.\mathrm{CO}\right)$, procesos de mejoramiento de la madera como biocombustible sólido, y la producción de biocarbón mediante un método alternativo a la pirólisis en atmósfera inerte. Por último, se revisa el potencial de mercado de la diversificación de productos forestales a través de los procesos anteriormente mencionados. Dado que tales procesos son de naturaleza termoquímica (incluyen gasificación y pirólisis), se utiliza la expresión "biorrefinería termoquímica" para diferenciarlos de procesos bioquímicos (fermentación y digestión anaerobia) comúnmente asociados a la biorrefinería (Demirbas et al., 2009; Vishnu \& Mala, 2012).

\section{BIORREFINERÍA TERMOQUÍMICA Y SU APLICABILIDAD EN COLOMBIA}

En su forma más simple, el concepto de biorrefinería es análogo al de las actuales refinerías de petróleo que producen múltiples combustibles y productos a partir del petróleo crudo, con la diferencia de que en las biorrefinerías se parte de materias primas biológicas. El concepto abarca una amplia gama de procesos y tecnologías, capaces de separar los recursos de biomasa (madera, pastos, maíz, semillas, etc.) en sus componentes básicos (carbohidratos, proteínas, triglicéridos, etc.), o en su forma elemental (carbono, hidrógeno, nitrógeno, etc.), para transformarlos en productos de valor agregado, como bioenergía, biocombustibles y productos químicos, los cuales se consideran soluciones alternativas al mundo postpetroquímico (Taylor, 2008). Los procesos más utilizados en el procesamiento de biomasa incluyen un enfoque metabólico o bioquímico para la producción de etanol, biocombustibles y bioproductos (Vishnu \& Mala, 2012), o la transformación termoquímica, que incluye procesos de gasificación y pirólisis (Linghong et al., 2010).

La investigación en biorrefinerías se ha intensificado en la última década debido, en parte, al agotamiento de los recursos fósiles, sobre los cuales está fundamentado el esquema energético mundial en la actualidad (Demirbas, 2009; Vishnu \& Mala, 2012). Por tal razón, se está investigando en diferentes frentes, como son la identificación de las materias primas más adecuadas, los fenómenos fisicoquímicos que tienen lugar durante la conversión, las diversas tecnologías existentes para llevar a cabo la síntesis, la optimización de la producción y las propiedades fisicoquímicas de las salidas del proceso, entre otras. En esta sección se presenta una revisión del estado actual de estas áreas del conocimiento en temas específicos de biorrefinería termoquímica y de los resultados obtenidos por diferentes autores en trabajos científicos.

\section{Biorrefinería}

Según Cherubini (2010) y Ghatak (2011), en el marco de las biorrefinerías, las principales fuentes de materias primas son las semillas dedicadas, residuos forestales y agrícolas, residuos de procesos industriales y domésticos, e incluso algas marinas. Los procesos termoquímicos (gasificación y pirólisis) y bioquímicos (fermentación y digestión anaerobia) son los utilizados usualmente, idea que es reiterada por Demirbas et al. (2009). Con respecto a los productos obtenidos, se destaca que no solamente son biocombustibles sólidos (pélets, lignina y biochar), líquidos (bioetanol, biodiésel, combustibles automoción y bioaceites) y gaseosos (biogás, biosyngás, hidrógeno y biometano), sino también materiales y productos químicos. Por ejemplo, los ácidos, polímeros, resinas y fertilizantes obtenidos de estos procesos son de gran utilidad en diversos sectores industriales (Cherubini, 2010; Demirbas, 2009). Por lo tanto, el reto de las biorrefinerías es desarrollar nuevas tecnologías, crear nuevos procesos y productos para asegurar el crecimiento sostenible desde lo económico, ambiental y social, con criterios de sostenibilidad (Cherubini, 2010; Demirbas et al., 2009). Demirbas et al. (2009) destacan la biomasa como materia prima para las biorrefinerías dada la protección del medio ambiente, ya que absorbe $\mathrm{CO}_{2}$ durante el crecimiento y lo 
emite durante la combustión. En este contexto, el concepto de biorrefinería es atractivo, ya que busca integrar la utilización de la biomasa con mínimos impactos ambientales (Ghatak, 2011).

Octave \& Thomas (2009) analizan varios aspectos inherentes al concepto de biorrefinería, como las diversas etapas del proceso y la gran diversidad de productos obtenidos y sus posibles usos industriales, incluyendo algunos poco tradicionales como el sector farmacéutico y cosmético, el sector alimenticio y la construcción. Estos autores consideran que procesar lignocelulosa mediante procesos termoquímicos o procesos bioquímicos en una biorrefinería es una alternativa muy interesante comparada con procesos que se basan en azúcar o en lípidos.

Los escenarios industriales más prometedores para los productos de biorrefinería son los que conciernen a las moléculas, los materiales y la energía. Las moléculas se pueden utilizar como tensoactivos de disolventes o productos químicos intermedios para la sustitución de derivados del petróleo, así como lubricantes; también se ha explorado la extracción de metabolitos secundarios de algunas especies y su uso como materia prima para síntesis químicas, fármacos y cosméticos. Por otro lado, las fibras pueden resultar valiosas en la producción de materiales compuestos; mientras que los azúcares y aceites actualmente se utilizan en la producción de biocombustibles, como bioetanol o biodiésel. No obstante, el desarrollo de técnicas eficientes para fraccionar la biomasa lignocelulósica en sus componentes básicos facilitará la investigación sobre la producción de sus derivados, tales como bioenergía, biomateriales y biocombustibles (Fitz Patrick et al., 2010).

En Colombia, la investigación en el tema de biorrefinerías ha venido aumentando en los últimos años, pero a nivel industrial, se puede decir que lo más semejante a una biorrefinería es lo que se ha hecho a partir de la caña de azúcar. En algunos ingenios azucareros del país se están obteniendo diversos productos con valor agregado a partir de esta materia prima, tales como azúcar, energía, papel, etanol, fertilizantes, entre otros (Dávila, 2013). Otras líneas de desarrollo en este ámbito están enfocadas al aprovechamiento de frutas, algas marinas, cultivos de palma de aceite, entre otros. Sin embargo, es poco lo que se ha trabajado y avanzado a partir madera forestal en nuestro país, con lo cual el futuro en esta línea de investigación y desarrollo resulta muy prometedor desde diferentes perspectivas: técnica, energética, social y ambiental.

\section{Gasificación de biomasa para bajas potencias}

Los procesos de gasificación de biomasa con fines energéticos se pueden hacer a nivel industrial y a baja-media escala, dependiendo de la demanda de energía que se tenga. Para cada caso se han encontrado tecnologías y procesos que son más eficientes que otros. Por ejemplo, en el caso de generación de energía a baja-media escala, se ha reportado que la mejor opción es la gasificación en lecho fijo (Di Blasi, 2000; IBRD, 1999). Además de estudios acerca de la tecnología y el proceso utilizado, son muchos los autores que se han dedicado a analizar el efecto que tienen diversas variables en el proceso de gasificación, tales como tipo de biomasa, propiedades físico-químicas de las maderas, relación combustible/aire, la temperatura de gasificación, y el agente gasificante, entre otros.

Lapuerta et al. (2008) estudian el efecto del tipo de biomasa y la relación combustible/aire (dosado) en el proceso de gasificación a presión atmosférica en lecho arrastrado a escala de laboratorio. La biomasa residual evaluada proviene del sector agrícola (uva y olivo) y del sector industrial (aserrín), las pruebas se llevan a temperatura constante $(1523 \mathrm{~K})$ y se varía el dosado entre 2 y 6 . Los resultados no muestran diferencias significativas entre los residuos de biomasa caracterizados, lo que indica que estos desechos podrían ser utilizados en los sistemas de modo flexible. El dosado es el principal parámetro que afecta la variación de la composición del gas y la eficiencia del proceso, ya que un aumento del dosado y/o la temperatura de 
reacción producen un incremento en la concentración de $\mathrm{H}_{2}, \mathrm{CO}$ y $\mathrm{CH}_{4}$. Asadullah et al. (2004) gasifican cuatro tipos de biomasa residual (cedro, yute, bagazo de caña y cascarilla de arroz) en un reactor de doble lecho fluidizado acoplado a un reactor catalítico. El objetivo es evaluar la conversión de carbón a gas en función del tipo de biomasa. Este tipo de gasificador aumenta la eficiencia de conversión a gas incluso a relativas bajas temperaturas, y alcanza el $80 \%$ de conversión a gas entre 823 y $923 \mathrm{~K}$. La eficiencia de conversión es afectada por la composición elemental de la biomasa, donde se obtiene un aumento de la conversión con el mayor contenido de carbón del biocombustible (cedro $>$ yute $>$ bagazo $>$ cascarilla de arroz). Por su parte, Hanaoka et al. (2005) caracterizan el proceso de gasificación en lecho fijo (1173 K y $1 \mathrm{~atm})$ de los diferentes componentes de la biomasa utilizando aire/vapor como agente gasificante. Se estudió la celulosa, xilano, lignina, quercus mongólica y pinus densiflora. Las conversiones a gas de la celulosa, xilano y lignina fueron del $97.9 \%, 92.2 \%$ y $52.8 \%$ sobre una base de carbono, respectivamente; mientras que la composición del biosyngás fue similar independiente de la materia prima utilizada. Lenis et al. (2013) caracterizan el proceso de gasificación de biomasa de cinco especies forestales en un reactor de lecho fijo a escala de laboratorio. Evalúan el efecto de las propiedades físico-químicas de las maderas (humedad, densidad aparente, poder calorífico y relación molar hidrógeno/carbono $(\mathrm{H} / \mathrm{C})$ ) sobre el proceso de gasificación. Los experimentos se llevaron a cabo utilizando el mismo tamaño de astilla, 4-10 mm, y con flujo de aire constante. Aunque las cinco especies de biomasa consideradas poseían similar composición química, según los respectivos análisis próximo y elemental, las diferencias en los resultados se deben a las variaciones en las propiedades físicas de cada especie, principalmente la densidad aparente.

Zhang et al. (2011) estudian la relación entre las eficiencias exergéticas y energéticas con la relación equivalente (RE) utilizando un modelo en equilibrio químico. Las eficiencias energéticas de todas las materias primas (madera, pino, arroz y polipropileno) estuvieron en el rango de $52.38 \%$ a $77.41 \%$, mientras que las eficiencias exergéticas alcanzaron valores entre $36.5 \%$ y $50.2 \%$. Ptasinski et al. (2007) desarrollan un análisis exergético del proceso de gasificación de diferentes tipos de biomasa (madera, aceite vegetal, lodos y estiércol) considerando el carbón como combustible de referencia. El estudio se llevó a cabo mediante un modelo en equilibrio desarrollado en Aspen Plus, y el valor promedio óptimo de la eficiencia exergética fue del $77 \%$. Sin embargo, se encuentra una mayor eficiencia exergética del carbón con respecto a la biomasa sólida, debido a la mayor temperatura de reacción del carbón (mayor exergía física). La gasificación de los aceites vegetales fue similar a la gasificación del carbón, y estos dos combustibles pueden ser considerados como combustibles de alta calidad.

Buragohain et al. (2010) determinan variables de optimización del proceso de gasificación de biomasa y del proceso de síntesis de FischerTropsch utilizando tres tipos de biomasas diferentes: aserrín, cascarilla de arroz y bagazo de bambú. El modelo matemático se desarrolla mediante la minimización de la energía libre de Gibbs, y el análisis considera el tipo de biomasa, la temperatura de gasificación $\left(400\right.$ a $1000^{\circ} \mathrm{C}$ ) y el agente gasificante (aire, mezclas aire-vapor). Las condiciones óptimas se encontraron con una RE entre 0.2 y 0.4 , y una temperatura entre 800 y $1000^{\circ} \mathrm{C}$. Según las condiciones con las que sale el gas de gasificación (relación $\mathrm{H}_{2} / \mathrm{CO}$ y el poder calórico (PC) del gas), este se puede utilizar en el proceso de síntesis de Fischer-Tropsch o en un sistema de potencia descentralizado.

Vaezi et al. (2012) desarrollan una metodología para la selección de materias primas de biomasa apropiadas para ser gasificadas. Para ello, desarrollan un algoritmo numérico que simula el proceso de gasificación de biomasa en equilibrio. La composición elemental de las biomasas simuladas comprendió un amplio rango de contenidos 
de oxígeno ( 27 a $44 \%$ en peso) y de relación carbono/hidrógeno (5 a 12), mientras que las demás condiciones operativas permanecieron constantes $(R E=0.4$, presión 1 atm y aire a $298 \mathrm{~K}$ como agente gasificante). Los resultados presentan las variables respuesta (eficiencia en frío, eficiencia de conversión de carbón, poder calorífico del syngás y temperatura) en función del contenido de oxígeno y la relación $\mathrm{C} / \mathrm{H}$ de la biomasa. Dicha información permite predecir las condiciones de operación del gasificador en función de un tipo de biomasa. Es así como un biosyngás con un PC de $370 \mathrm{MJ} / \mathrm{kmol}$ y una temperatura de $1200 \mathrm{~K}$ corresponde a una biomasa con contenido de oxígeno entre 30 y $39 \%$ wt y una relación $\mathrm{C} / \mathrm{H}$ de 7.9 a 8.5. Sin embargo, la temperatura restringe el contenido de oxígeno a 30-35\%.

Cuando se piensa en la aplicabilidad de gasificación de biomasa con fines energéticos en Colombia, se encuentra que, debido a la gran disponibilidad de recursos hídricos en el país, solo se considera el potencial de la biomasa como fuente no convencional de energía en ZNI. Estas regiones no interconectadas corresponden al 52\% del territorio nacional y son habitadas por cerca del 12\% de la población Colombiana (UPME, 2010c). Según los últimos reportes de la UPME, la generación de energía con biomasa representa solo el $2.78 \%$ de la capacidad efectiva de generación en Colombia (Figura 2).

Teniendo en cuenta la baja densidad poblacional de las ZNI, la demanda energética localizada estaría en promedio por debajo de los 500 kWe. Para esta escala de generación, se ha identificado que la mejor opción en cuanto a tecnologías que operan con biomasa es la gasificación en lecho fijo (Di Blasi, 2000; IBRD, 1999), pues permite el uso de la biomasa en el mismo lugar en que se genera, y disminuye los costos asociados a su almacenamiento y transporte, los cuales son uno de los principales retos que afronta el aprovechamiento energético de biomasa (Melgar et

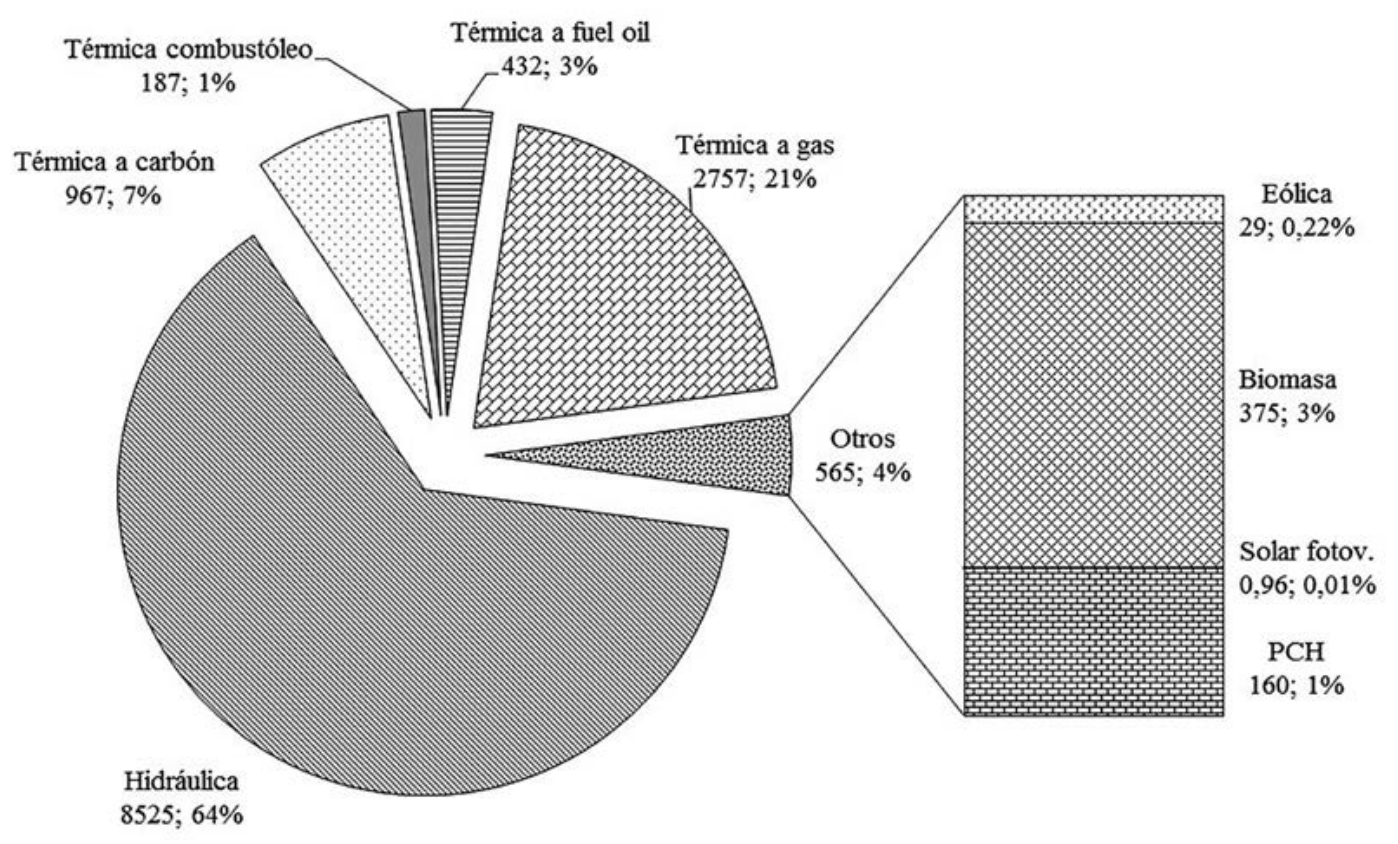

Figura 2. Capacidad de generación instalada efectiva [MW] en Colombia, adaptado de (UPME, 2009). 
al., 2008). Según estudios realizados por la UPME (2010a), para tecnologías de gasificación se pueden tener costos de generación de alrededor de 200 COP/kWe-h (Figura 3), los cuales están por debajo de los obtenidos para plantas diésel, que son las tecnologías utilizadas convencionalmente para generación en ZNI.

Relacionando las ZNI con el PND, es necesario establecer criterios de selección energética de las especies forestales para generación de energía descentralizada. En un trabajo reciente, Osorio et al. (2014) definen criterios silviculturales para la selección de maderas como combustible para programas de biopotencia a baja-media escala. Los autores concluyen que algunas de las variables silviculturales más importantes son el área plantada, el índice medio anual y el turno biológico, criterios que resultan de gran importancia al momento de planear un programa de gasificación de biomasa con fines energéticos en el país.

\section{Biocombustibles gaseosos de segunda generación}

La gasificación de madera en los reactores de flujo arrastrado produce gas de síntesis (biosyngás), que es una mezcla compuesta principalmente por hidrógeno y monóxido de carbono (Hook \& Aleklett, 2010; Robinson \& Luyben, 2008). Las necesidades urgentes para la producción de combustibles y productos químicos a partir de gas de síntesis impulsan el estudio de este proceso termoquímico (Hook \& Aleklett, 2010; Robinson \& Luyben, 2008; Zhao et al., 2006). Unidades de gasificación en las centrales eléctricas producen un gas combustible para mover las turbinas de gas. La gasificación en plantas químicas produce gas de síntesis que se pueden utilizar para producir un amplio espectro de productos químicos, tales como amoníaco, metanol, metano y combustibles líquidos (Gasifipedia, 2013; Hook \& Aleklett, 2010). Se prevé que

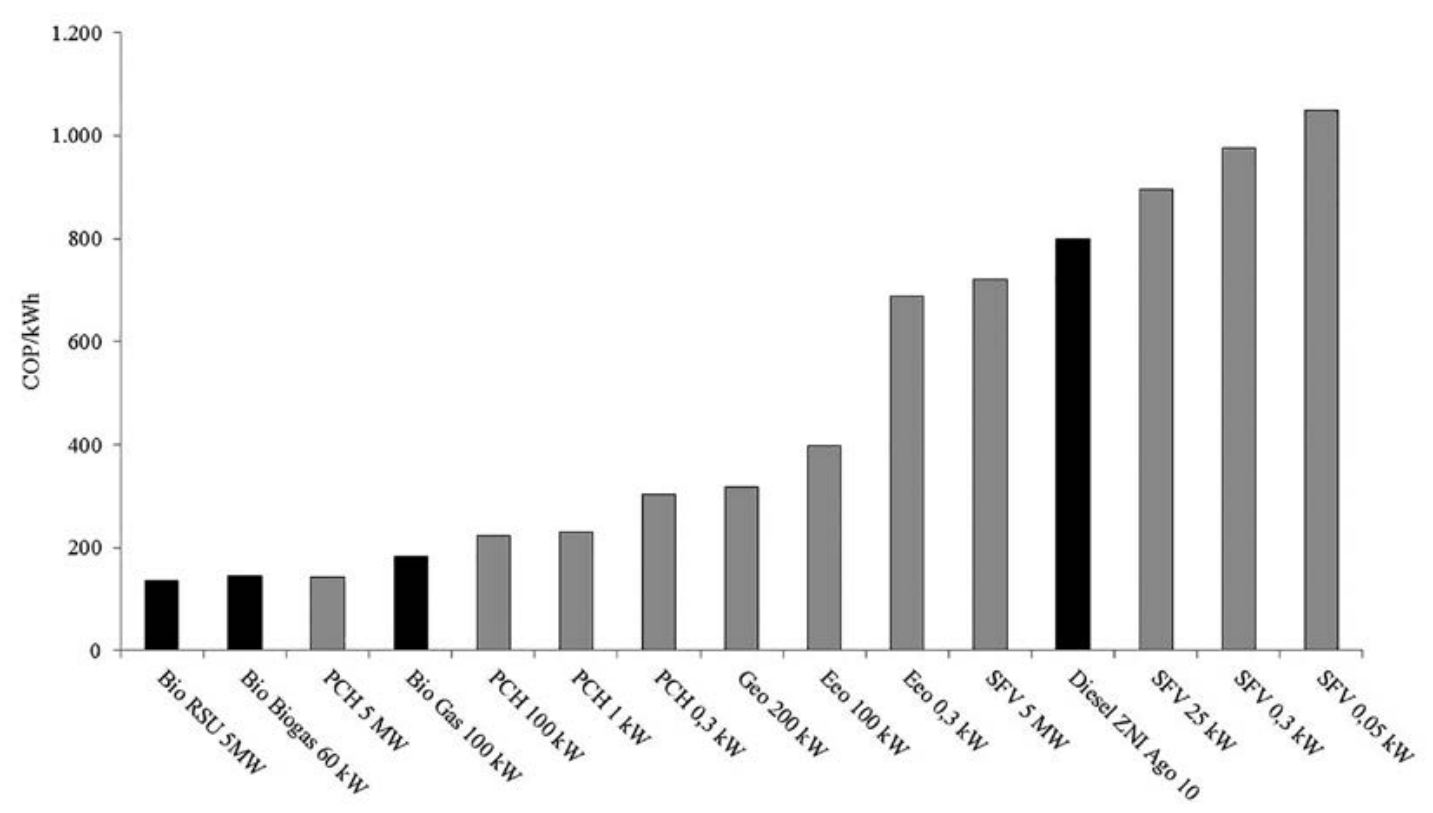

Figura 3. Costos de generación de las FNCE en ZNI colombianas. Adaptado de UPME (2010c). Abreviaturas: COP: peso colombiano, Bio: biodigestores, RSU: residuos sólidos urbanos, PCH: pequeña central hidroeléctrica, Gas: gasificación, Geo: geotérmica, Eeo: energía eólica, SFV: solar fotovoltaica. 
las plantas químicas del futuro serán plantas híbridas de energía/químicos con la gasificación como unidad fundamental. Por lo tanto, la gasificación sigue siendo un tema importante para la investigación (Gasifipedia, 2013; Hook \& Aleklett, 2010; IEA, 2003; Robinson \& Luyben, 2008).

La producción de gas natural sintético (GNS) puede darse a través de la gasificación de carbón o de biomasa. Si bien históricamente el carbón ha sido una importante fuente de energía, representando aproximadamente el $25 \%$ de los suministros mundiales y $\sim 40 \%$ en la generación mundial de electricidad, y se prevé que siga desempeñando un papel importante en el cumplimiento de las crecientes demandas de energía del mundo (Hook \& Aleklett, 2010), el uso del carbón se enfrenta a desafíos como los sistemas de energía limpia y eficiente, el almacenamiento y secuestro de carbono, y el impacto ambiental debido a la explotación minera (Hook \& Aleklett, 2010; Lu et al., 2008). Por tal razón, la producción GNS a partir de biomasa cobra importancia, y es un proceso de especial interés en países como Colombia, donde se prevé el agotamiento del gas natural en los próximos años debido a la creciente demanda (UPME, 2010b). Por lo tanto, el proceso de gasificación de biomasa puede dar respuesta a uno de los principales retos que enfrenta el país en encontrar la forma de agregar valor a la madera comercial mediante criterios medioambientales, de eficiencia y sostenibilidad (DNP, 2010). A diferencia de la biomasa, la gasificación a escala industrial de carbón para producción de combustibles tales como GNS, hidrógeno, energía mediante gasificación integrada a ciclo combinado (GICC), entre otros, ha sido ampliamente estudiada (Kunze \& Spliethoff, 2011; Maurstad et al., 2013; Vitasari et al., 2011; Yu et al., 2012).

En este tema se encuentran trabajos de investigación con diferentes enfoques. Por ejemplo, Consonni et al. (2009) analizan la integración de una biorrefinería de celulosa utilizando licor negro (un subproducto de la extracción de la fibra de la madera, rico en lignina) y residuos de madera, con una fábrica papelera. El estudio incluyó simulaciones detalladas de balance de masa-energía y análisis financieros, energéticos y ambientales, para siete escenarios de operación de la planta. Tres escenarios enfocados en la producción de Dimetileter (aditivo para gasolina), otros tres en la producción de combustibles Fischer-Tropsch y uno en la obtención de alcoholes mixtos. El proceso incluye cinco etapas básicas: gasificación del licor negro, gasificación de la biomasa, recuperación de calor y limpieza del gas de síntesis, depuración del combustible, y finalmente la etapa de generación de potencia. El gasificador empleado es del tipo de flujo arrastrado, operando a 35 bar, $1000{ }^{\circ} \mathrm{C}$. La biorrefinería basada en gasificación de pulpa ofrece una atractiva posibilidad de revitalización de la industria de la pulpa y papel, al igual que brinda la posibilidad de ahorro energético (petróleo), disminuyen las emisiones, mejora la seguridad energética y el desarrollo económico rural.

Bludowsky \& Agar (2009) comparan diferentes rutas para obtención de metanol a partir de biogás sintético (biosyngás) con respecto a las demandas energéticas y proponen un novedoso concepto de integración energética. Los autores proponen la producción de biosyngás en una biorrefinería con niveles de temperatura inferiores a la temperatura convencional de los procesos de gasificación, lo que permite una integración térmica con los procesos exotérmicos aguas abajo, tales como la síntesis de metanol. Sin embargo, previo a su desarrollo a escala industrial, se deben aclarar los riesgos considerables que aún plantea el funcionamiento a largo plazo de etapas posteriores requeridas en el proceso, como el reformado en fase acuosa con la reacción agua-gas (water gas shift reaction). Tay et al. (2011), mediante un enfoque de optimización modular, integran un modelo de gasificación de biomasa (basado en equilibrio químico) y modelos estructurales de los procesos de síntesis para dos casos de estudio. El objetivo es evaluar la composición de equilibrio de gas de síntesis, la temperatura óptima de funcionamiento y los tipos y cantidades requeridas de oxidantes. Los análisis demuestran 
que los precios de dimetiléter, combustible-alcohol mezclado y biosyngás deben tenerse en cuenta en las fases avanzadas de la síntesis de una biorrefinería integrada basada en gasificación, con el fin de asegurar su viabilidad económica.

Kumar et al. (2009) presentan una revisión sobre la gasificación de la biomasa para producción de biocombustibles, bioenergía y productos químicos. Según los autores, entre las diferentes alternativas de conversión de energía, la gasificación de biomasa cuenta con un gran potencial debido a la flexibilidad de uso de materias primas. Sin embargo, los desafíos técnicos en la comercialización de biocombustibles y productos químicos a partir de la biomasa incluyen el aumento de la eficiencia energética del sistema, el desarrollo de tecnologías robustas y eficientes para la limpieza del biosyngás y su conversión en combustibles y productos químicos valiosos.

\section{Biocarbón}

El carbón activado (CA) es reconocido como material poroso de alta área superficial y relativa alta resistencia mecánica. El tamaño y distribución de poros lo hace útil para absorción de gases y líquidos. Por tal razón, ha sido ampliamente utilizado en separación de gases, control de emisiones, recuperación de solventes, remoción de contaminantes orgánicos de agua potable, tratamiento de aguas residuales y soporte catalítico (Adinata et al., 2007; Guo \& Lua, 1998). El carbón se obtiene a partir de fuentes como petróleo, brea, turba, polímeros, carbón mineral o maderas, entre otros, y posteriormente se somete a procesos de activación (eliminación de volátiles, aumento de volumen de poros y superficie específica) a través de procesos físicos (carbonización a temperaturas cercanas a los $1000{ }^{\circ} \mathrm{C}$ ) o procesos químicos (impregnación con ácidos a temperaturas cercanas a los $600^{\circ} \mathrm{C}$ ). No obstante, diversos investigadores han explorado las propiedades de biocarbones (biochar) producidos a partir de la biomasa mediante diferentes metodologías para usos y aplicaciones similares a las del CA. Además, el biocarbón puede añadirse a los suelos con el objetivo de fertilizarlos y de mejorar sus funciones, o para reducir las emisiones de la biomasa que de otro modo se degradaría naturalmente a gases de efecto invernadero. El biochar también tiene un valor apreciable como secuestrador de carbono. Estas propiedades son medibles y verificables en un esquema de caracterización, o en un protocolo de compensación de emisiones de carbono (Adinata et al., 2007; Guo \& Lua, 1998).

En la literatura científica se encuentran diversos trabajos que abordan esta temática, tratando principalmente de determinar las características y propiedades fisicoquímicas del biochar y de las variables más influyentes en su formación. Kwapinski et al. (2010) determinan las propiedades físicas y la composición del biochar producido a partir de la descomposición térmica del sauce y el pino, a temperaturas entre 400 y $600{ }^{\circ} \mathrm{C}$. Para ello partieron de muestras de $250 \mathrm{~g}$, astilladas a un tamaño máximo de $1 \mathrm{~cm}$, y calentadas a 400, 500 y 600 ${ }^{\circ} \mathrm{C}$, en ensayos diferentes, durante 10 minutos en un pirolizador en ausencia de aire. A los productos obtenidos se le realizaron análisis elementales, de área superficial y de poder calorífico, y con ellos se pudo establecer que al aumentar la temperatura, la producción de biochar disminuye, mientas que la cantidad de carbono aumenta, y el poder calorífico no varía significativamente. Anderson et al. (2013) efectúan una caracterización, en relación con las propiedades químicas, posibles usos y mercados, del gas sintético, biochar y del carbón activado producidos en una planta piloto de gasificación y otra de pirólisis. Utilizan como materia prima astillas de coníferas y residuos forestales, con tamaño de partícula similar, pero diferente contenido de carbono. La composición del gas se analizó mediante cromatografía gaseosa, y la activación del biochar se efectuó a $950{ }^{\circ} \mathrm{C}$ utilizando vapor. En cuanto al biochar, para las dos materias primas utilizadas se observó que el tamaño de partícula se distribuyó en torno a $0.84 \mathrm{~mm}$, con densidad entre 131 y $183 \mathrm{~kg} / \mathrm{m}^{3}$, $\mathrm{PH}$ entre 8.7 y 10.2 , 
contenido de carbono entre 70 y $90 \%$, y número de yodo entre 245 y $425 \mathrm{~g} / \mathrm{cg}$, indicando una alta capacidad de adsorción. En términos generales, los autores concluyen que los principales obstáculos para una integración y distribución a gran escala de estas tecnologías corren más por cuenta de aspectos financieros que técnicos.

Qian et al. (2013) estudian el efecto del tipo de biomasa y la relación aire/combustible (RE) sobre las propiedades fisicoquímicas del char obtenido en procesos de gasificación. Para tal fin llevaron a cabo diversos experimentos en un gasificador a escala de laboratorio de lecho fluidizado. El reactor fue alimentado con tres tipos de biomasa astillada a un tamaño de $1.25 \mathrm{~cm}$. Se evaluaron tres RE, $0.20,0.25$ y 0.28 , con flujos másicos de 2 a $5 \mathrm{~kg} / \mathrm{h}$. Las temperaturas promedios de gasificación fueron de 700,780 y $800{ }^{\circ} \mathrm{C}$ para cada RE, con tiempos de residencia de alrededor de $7 \mathrm{~s}$. Tanto la biomasa utilizada, como el char obtenido, fueron objeto de análisis próximo y elemental, área superficial y morfológico con SEM. Los resultados permitieron establecer que el área superficial del char se encontró entre 1 y $10 \mathrm{~m}^{2} / \mathrm{g}$, observándose una dependencia con la RE. Por su parte, el contenido de ceniza en el char fue alrededor de $40 \%$, bastante mayor que el de la biomasa (5\%). Lo anterior presentaría inconvenientes en la utilización del char como precursor del carbón activado o como combustible. El análisis FT-IR mostró que, durante la gasificación, las materias primas pierden enlaces alifáticos $\mathrm{CH}$, pero mantienen enlaces $\mathrm{CH}$ aromáticos en el char. Además, los enlaces C-O-C del char son más débiles que los de la biomasa, lo que indica la fuerte descomposición de la celulosa y la hemicelulosa.

Molina (2012) realiza un estudio experimental de caracterización estructural y termoquímica del char producido en un gasificador de biomasa en lecho arrastrado a presión atmosférica. Analiza los cambios físicos y químicos que tienen lugar durante el proceso de conversión de la biomasa sólida a gas, y propone posibles aplicaciones del residuo carbonoso. En este estudio se utilizaron residuos agrícolas e industriales, y el biochar se analizó mediante análisis inmediato, análisis termogravimétrico (TGA), FTIR, estructural y morfológico, cuyos resultados indican la aplicabilidad del char más como fertilizante y acondicionador del suelo que como carbón activado. También se encontró que para una relación combustible/aire próxima a 3.5, se maximiza la superficie específica del char, pero disminuyen la reactividad, el poder calorífico y los grupos funcionales.

Márquez-Montesino et al. (2013) estudiaron la capacidad de adsorción de amoniaco, benceno y gasolina en carbones activados obtenidos a partir de residuos de tomate y considerando la temperatura de activación, la concentración de la disolución ácida y la relación de impregnación (RI) como variables experimentales. Tras caracterizar la materia prima mediante análisis inmediato y determinar el número de yodo, se activó el carbón para la adsorción de vapores a temperaturas de 400 y $500{ }^{\circ} \mathrm{C}$, con $\mathrm{RI}$ de 10, 25 y 40 . Los resultados indicaron que para el amoniaco, la temperatura de activación, la concentración de la disolución ácida y la interacción temperatura de activación-RI fueron significativamente influyentes en la capacidad de adsorción, caso contrario al benceno, en donde no se observó una dependencia significativa. Para la gasolina, la concentración de la disolución ácida y la interacción temperatura de activación-RI resultaron ser los más influyentes sobre la adsorción, y se aclara que en todos los casos la capacidad de adsorción se vio favorecida con el incremento de la temperatura de activación y de la RI.

Por su parte, San Miguel et al. (2012) caracterizan las partículas sólidas en el gas producido en una planta de gasificación operando con madera de pino, y evalúan los riesgos para el ser humano y el medio ambiente. La experimentación se Ilevó a cabo en una planta que a plena carga consume $800 \mathrm{~kg} / \mathrm{h}$ de biomasa de pino, con un tamaño menor a $10 \mathrm{~cm}$, y que produce un gas sintético con poder calorífico de $4.6 \mathrm{MJ} / \mathrm{Nm}^{3}$ y con un contenido de $50 \mathrm{mg} / \mathrm{Nm}^{3}$ de partículas sólidas, que representan cerca del $6 \%$ en peso de la biomasa alimentada. A estas partículas en el biosyngás se 
les practicaron análisis de composición elemental, análisis morfológicos con SEM, termogravimetría, textura y adsorción, de composición de inorgánicos por inducción con plasma, y presencia de hidrocarburos poliaromáticos $(\mathrm{PAH})$ con cromatografía gaseosa. Los resultados de estos análisis permitieron concluir que la aparición de partículas sólidas durante la gasificación se debe al alquitrán formado por la condensación y carbonización de la biomasa. Estructuralmente, la baja área superficial, la baja capacidad de adsorción y la naturaleza heterogénea a nivel microscópico de las partículas las hacen poco prácticas para su uso como catalizadores o en aplicaciones de tratamiento de aguas o gases. Por otra parte, la baja proporción de partículas con tamaño menor a $10 \mathrm{~mm}$, así como de $\mathrm{PAH}$, minimiza los riesgos a la salud humana. No obstante, debido a la presencia de nutrientes y micronutrientes y a la ausencia de materiales tóxicos, podrían utilizarse como fertilizantes o acondicionadores del suelo.

Tal como se evidencia en la anterior revisión, el biocarbón obtenido como producto de valor agregado en una biorrefinería sería de gran aplicabilidad en Colombia, bien sea para uso como fertilizante, como secuestrador de carbono, para tratamiento de aguas residuales, entre otros.

\section{Torrefacción como pretratamiento para mejorar la calidad de la madera como biocombustible sólido}

La torrefacción es un proceso de pirólisis atenuada, que se lleva a cabo en el intervalo de temperatura de 200 a $300{ }^{\circ} \mathrm{C}$, y ha sido considerado como una vía eficaz para mejorar las propiedades de la biomasa y aumentar su densidad energética. En la actualidad, es una tecnología que está siendo considerada como una de las mejores opciones para la producción de vectores bioenergéticos sólidos a escala piloto e industrial (Phanphanich \& Mani, 2011). Van der Stelt et al. (2011) afirman que la torrefacción tiene un gran potencial para convertirse en la tecnología de pretratamiento de la biomasa, de cara a lo que es la obtención de productos sólidos con muy buenas características y propiedades combustibles, como la densidad energética, homogeneidad, triturabilidad y comportamiento hidrofóbico. Prins et al. (2006) comparan exergéticamente el proceso de gasificación de la madera torrefactada con respecto a la madera sin pretratar, utilizando aire y oxígeno como agente gasificante por separado. El estudio se realizó mediante un modelo en equilibrio desarrollado en Aspen Plus, y encontró que al utilizar un proceso de torrefacción $\left(250-300{ }^{\circ} \mathrm{C}\right)$ de la madera como pretratamiento se reducen las pérdidas termodinámicas, es decir, las irreversibilidades. Según los resultados, la torrefacción es un método prometedor para lograr mayor eficiencia en la gasificación.

Ciolkozs \& Wallace (2011) revisan el proceso de torrefacción de biomasa para la producción de bioenergía. Destacan la mayor triturabilidad y resistencia a la humedad de la biomasa torrefactada como las dos características que le dan más valor a este pretratamiento. Sin embargo, aclaran que la compleja naturaleza química del proceso amerita un mayor entendimiento y estudio, especialmente en lo que respecta a la composición química del char sólido producido, así como los riesgos a la salud que podría representar la operación a escala global. Dada la importancia del tema, muchos trabajos científicos han venido abordando de forma experimental o por simulación los aspectos inherentes a este mejoramiento de la calidad de la biomasa como biocombustible sólido.

Bridgeman et al. (2010) estudian el efecto de la temperatura, tiempo de residencia y tamaño de partícula, bajo cuatro condiciones de torrefacción en el grado de trituración de dos semillas (Sauce y Miscanthus). La biomasa y productos torrefactados se caracterizaron mediante análisis próximo y elemental, y el grado de trituración de los productos se estableció con base en el HGI (Hardgrove Grindability Index) para carbones duros. El estudio se llevó a cabo mediante un diseño multifactorial con tres factores: 230,250 y $290{ }^{\circ} \mathrm{C}$ para la temperatura; 10 min y 60 min de tiempo de residencia; 
y $4 \mathrm{~mm}, 10 \mathrm{~mm}$ y $20 \mathrm{~mm}$ de tamaño de partícula. Los resultados muestran que el orden de importancia en el contenido de carbón (contenido de energía) es temperatura, tiempo de reacción y tamaño. El Sauce requiere altas temperaturas y tiempos de residencia más largos a fin de obtener un comportamiento similar al del carbón, mientras que el Miscanthus requiere un tratamiento a temperaturas en torno a $290{ }^{\circ} \mathrm{C}$, pero con tiempos de residencia menores.

Chen \& Kuo (2010) estudian la torrefacción de cuatro especies de biomasa (bambú, sauce, coco y una madera nativa) mediante análisis de TGA, enfocándose en el efecto del contenido de celulosa y hemicelulosa de las biomasas. Se consideraron dos temperaturas de torrefacción, 240 y $275{ }^{\circ} \mathrm{C}$, donde la menor temperatura tuvo un efecto significativo sobre el consumo de la hemicelulosa, pero su impacto en la celulosa y la lignina fue mínimo. Concluyeron que la torrefacción es considerada como una operación apropiada de pretratamiento de la biomasa en la producción de combustibles. En un trabajo posterior, Chen \& Kuo (2011) tratan de predecir la descomposición térmica de algunos constituyentes de la biomasa, como la hemicelulosa, celulosa, lignina y xilano mediante un modelo cinético basado en termogravimetría (TGA). El calentamiento consistió en tres períodos dinámicos $\left(20^{\circ} \mathrm{C} / \mathrm{min}\right)$ y dos isotérmicos, el primero hasta llegar a $105^{\circ} \mathrm{C}$ y el segundo hasta llegar a la temperatura de torrefacción según el caso $(200,225,250$, 275 y $\left.300{ }^{\circ} \mathrm{C}\right)$, manteniéndola por espacio de una hora. Posteriormente se continuó el calentamiento a la misma velocidad hasta alcanzar la pirólisis a unos $800^{\circ} \mathrm{C}$. Se encontró que el xilano resultó ser la especie más sensible a la torrefacción, especialmente en el rango de temperatura de 200 a $300{ }^{\circ} \mathrm{C}$. Del estudio cinético realizado, se encontró que la energía de activación de la hemicelulosa, celulosa, lignina y xilano son 187.06, 124.42, 37.58 y $67.83 \mathrm{~kJ} / \mathrm{mol}$, respectivamente.

Esteves et al. (2013) estudian la influencia de la torrefacción (180 y $210{ }^{\circ} \mathrm{C}$ ) en los cambios en la estructura y composición química de dos maderas (blanda y dura). Los ensayos se llevaron a cabo durante tiempos de 2, 6, 12 y 24 horas, en lotes de 4 muestras por cada tiempo de residencia. Según el estudio, se pudo establecer que la hemicelulosa es el primer componente en degradarse por rotura de las cadenas laterales alifáticas, siendo más evidente en la madera dura que en la blanda. Sin embargo, los cambios inducidos por el tratamiento térmico son difíciles de monitorear mediante espectroscopia con FTIR, debido a que las diferentes reacciones químicas ocurren simultáneamente.

Chaouch et al. (2013) estudian el efecto de la intensidad de la torrefacción sobre variables como la composición elemental, la durabilidad, la eficiencia de antiinflamación (dilatación volumétrica) y el contenido de humedad. Analizan varias especies de maderas europeas, tanto duras como blandas a $230{ }^{\circ} \mathrm{C}$ en atmósfera inerte y con varios tiempos de duración. Los resultados del estudio confirman que todas las nuevas propiedades adquiridas por la madera después del tratamiento térmico dependen del nivel de termodegradación (intensidad del tratamiento). La durabilidad, el contenido de humedad y el contenido de oxígeno disminuyen progresivamente a medida que aumenta la intensidad del tratamiento, mientras que la eficiencia de antiinflamación y el contenido de carbono aumentan.

En la misma dirección, Phanphanich \& Mani (2011) investigan las características y la triturabilidad de astillas de pino y residuos de tala de madera. La torrefacción se llevó a cabo en un horno eléctrico con atmósfera inerte $\left(\mathrm{N}_{2}\right)$, se utilizaron entre 1 y $1.5 \mathrm{~kg}$ de biomasa a temperatura ambiente, y se calentó a una razón de $10^{\circ} \mathrm{C} / \mathrm{min}$ hasta alcanzar la temperatura de torrefacción 225, 250, 275 y $300{ }^{\circ} \mathrm{C}$, la cual se mantuvo por 30 minutos. A los productos torrefactados se les determinó el tamaño y distribución de partículas, análisis de densidad, análisis próximo y elemental. Los resultados indicaron que la torrefacción de astillas de madera limpia y de residuos de la tala maderera mejora significativamente el poder calorífico, en comparación con la biomasa sin tratar. 
Chen et al. (2011) caracterizan el efecto de la torrefacción de madera (madera de Lauán) en las propiedades de los productos y en la formación de condensados. Para tal fin experimentan con tres temperaturas, $200{ }^{\circ} \mathrm{C}$ (torrefacción ligera), $250{ }^{\circ} \mathrm{C}$ (torrefacción media) y $280{ }^{\circ} \mathrm{C}$ (torrefacción severa), así como cuatro tiempos de proceso, $0.5,1$, 1.5 y $2 \mathrm{~h}$. Con el aumento de la temperatura o el tiempo de torrefacción, aumenta el contenido de carbono y el poder calorífico de la biomasa. Sin embargo, a la temperatura de torrefacción de 280 ${ }^{\circ} \mathrm{C}$, la pérdida de peso de la madera fue drástica (> $50 \%$ ) con un aumento de poder calorífico mayor al $40 \%$. Con respecto a los condensados, más del $50 \%$ de la madera consumida se transforma en líquido, independiente de la temperatura y el tiempo del proceso. Los principales componentes de los condensados son monoaromáticos y pequeñas cantidades de hidrocarburos policíclicos, lo cual implica que deben ser tratados más como productos químicos que como combustibles.

Las propiedades mecánicas de la madera son estudiadas por Ghalehno \& Nazerian (2011), quienes analizan el efecto de la torrefacción sobre las propiedades físicas y mecánicas (densidad, dilatación volumétrica, ensayos mecánicos de compresión, flexión y dureza) de una madera nativa. Se estudiaron nueve grupos, cada uno con nueve muestras, además de un grupo adicional que sirvió de grupo de referencia. Las muestras de madera fueron sometidas a un tratamiento térmico a temperaturas de 130,160 y $190{ }^{\circ} \mathrm{C}$, durante periodos de tiempo de 3, 6 y 9 horas. La menor disminución en las propiedades mecánicas se observó en el tratamiento térmico de $130{ }^{\circ} \mathrm{C}$ durante $3 \mathrm{~h}$. También se observó un aumento de la estabilidad dimensional, lo cual es requerido en varias aplicaciones de la madera, esto puede explicarse por la degradación de la hemicelulosa, debido a la alta temperatura aplicada.

Ibrahim et al. (2013) caracterizan las propiedades fisicoquímicas del material torrefactado (capacidad de trituración, densidad, hidrofobicidad y área superficial). Los autores estudian varias especies de maderas (sauce, eucalipto, una mezcla de maderas de roble y abedul, y una mezcla de maderas blandas de pino alerce y abeto), a 270 y $290{ }^{\circ} \mathrm{C}$, con dos tiempos de residencia $(30$ y 60 min). A partir de los resultados se observó que el eucalipto experimenta la mayor pérdida de masa, aunque el sólido resultante tenía el mayor poder calórico de todas las biomasas estudiadas. Al eucalipto le siguieron la mezcla de maderas duras, seguido por el sauce, y finalmente la mezcla de maderas blandas. Los análisis químicos del sólido torrefactado indican que las relaciones de $\mathrm{O} / \mathrm{C}$ y $\mathrm{H} / \mathrm{C}$ disminuyen durante la torrefacción, por la pérdida de grupos $\mathrm{OH}$ y un aumento en los grupos $\mathrm{C}=\mathrm{O}$ en relación con los grupos $\mathrm{C}-\mathrm{O}$. El resultado es un material torrefactado más hidrófobo y más fácilmente triturable.

Por su parte, Esteves et al. (2011) comparan el proceso de torrefacción convencional con un proceso de calentamiento con vapor, con base en algunas de las propiedades fisicoquímicas de la madera de pino marítimo. El tratamiento térmico de la madera disminuye el contenido de humedad, la anisotropía y la humectabilidad de la superficie, mientras que la estabilidad dimensional aumenta. En relación con las propiedades mecánicas, el módulo de elasticidad no se vio afectado, pero la resistencia a la flexión disminuyó. Tal reducción se debió a las reacciones de oxidación y a la mayor degradación de la hemicelulosa. La pérdida de masa del pino tratado al calor del horno fue mayor que para el tratamiento con vapor. Para la misma pérdida de masa, el contenido de humedad disminuyó más con el tratamiento con vapor, debido a la mayor degradación de la hemicelulosa y celulosa.

Dadas las perspectivas de crecimiento del sector forestal comercial en Colombia, una de las posibles alternativas de mercado para la madera es su exportación como biocombustible sólido. Para ello es necesario asegurar ciertas especificaciones como contenido energético, humedad, reactividad bajo procesos termoquímicos, entre otros, algunos de los cuales pueden mejorarse a través de pretratamientos como la torrefacción. 


\section{POTENCIAL DE MERCADO}

En la actualidad, los bosques a nivel mundial ocupan cerca de 4.000 millones de hectáreas, que representan el $31 \%$ de la superficie del planeta (FAO, 2012). De esta superficie de bosque, se estima que el $95 \%$ corresponde a bosques naturales y el otro $5 \%$ a plantaciones forestales. La alta y creciente demanda de productos forestales ha conseguido que esta sea atendida tanto con bosques naturales como por plantaciones sembradas con fines económicos. Afortunadamente, las presiones ambientalistas hacen que cada vez sea menor la extracción de madera de los bosques naturales, lo que ayuda a su conservación. De ahí la reciente tendencia mundial a aumentar las plantaciones comerciales y a depender de ellas en mayor medida como fuente de madera industrial (Agrocadenas, 2005).

Este sector industrial, muchas veces inadvertido por la sociedad, es una fuente importante de empleo y bienestar. La industria forestal a nivel mundial aportaba, en el 2006, cerca del 1\% del PIB y generaba 13.7 millones de puestos de trabajo, que representa el $0.4 \%$ de la mano de obra total. Sin embargo, es importante anotar que hay regiones donde esta actividad económica es más representativa en generación de PIB y empleo, entre las que se encuentra Latinoamérica y el Caribe (FAO, 2008).

En este mercado se transan diferentes tipos de productos, desde materia prima (madera en rollo industrial y combustible de madera o leña) hasta productos terminados. Los productos terminados tradicionales son: madera aserrada para la construcción, fabricación de barcos, muebles, traviesas, etc.; trozas empleadas en la fabricación de distintos tipos de tableros de madera; puntales para usos en minería; pasta de madera para la producción de papel y cartón; entre otros. Sin embargo, en los últimos años la cadena productiva de la madera ha comenzado a evolucionar, buscando nuevos usos, nuevos productos y mercados diversificados (Chambost \& Stuart, 2007). Ahora se habla de productos con valor agregado como biocombustibles, bioenergía, bioproductos, biomateriales, combustibles de segunda generación, entre otros. Así, un estudio reciente realizado en Canadá estima que este mercado emergente en la industria forestal ofrece oportunidades hasta por 200 billones de dólares (FPAC, 2011).

Hoy en día, la madera resulta ser la fuente más importante de energía renovable en el mundo y para que sea una fuente más limpia se debe asegurar que la siembra sea en plantaciones sostenibles y la quema se realice con técnicas eficientes y con el mínimo de emisiones (FAO, 2012). En cuanto al consumo futuro de bioenergía, Buongiorno et al. (2011) tratan de determinar las consecuencias que traería para las reservas mundiales de bosque una rápida aceleración de la demanda de bioenergía. En este escenario, el consumo mundial de biocombustibles crecería un 80\% entre el año 2006 y el 2030, y provocaría un aumento en el precio mundial de la madera en rollo (30\%) y de la leña para generación de energía (144\%), precios que convergerían en el 2030. Por su parte, la producción mundial de leña aumentaría un $48 \%$, mientras que la producción mundial de madera en rollo disminuiría un 3\%, lo cual refleja la reasignación del mercado destino de la madera. Finalmente, según el autor, en este escenario, la reserva forestal global disminuiría un $2 \%$ en el 2030.

Para analizar la situación de Colombia en el mercado forestal internacional, es importante anotar que el $48 \%$ de la superficie del país (55 millones de hectáreas aproximadamente) corresponde a bosques naturales y plantaciones forestales, aunque al considerar restricciones de aptitud de uso, ecológicas y de accesibilidad, el área aprovechable se reduce considerablemente. La producción de madera en el país se basa principalmente en la explotación de bosques naturales, pues las plantaciones forestales apenas representan el 3\% del total de bosques colombianos, lo que muestra el déficit existente en plantaciones para fines económicos (Agrocadenas, 2005). La participación del sector productivo en la economía del país ha representado cerca del $0.14 \%$ del PIB nacional, porcentaje significativamente menor al del sector a nivel mundial. 
En vista de la escasez de plantaciones forestales, Proexport (Ilamada ahora Procolombia) está liderando una campaña de promoción con el objetivo de atraer inversionistas al sector forestal. Son muchas las ventajas y oportunidades que existen: Colombia tiene 17 millones de hectáreas con aptitud forestal y apenas se está usando el $2 \%$ de ellas; la localización en la zona tropical y los desarrollos en biotecnología hacen que el rendimiento y crecimiento de varias especies forestales sean mejores que en muchos otros países; la balanza comercial colombiana en madera, carbón vegetal y manufacturas de madera es deficitaria, lo que hace necesaria la importación de algunos productos; la tasa de retorno en inversiones forestales es atractiva frente a los competidores de la región; existen beneficios tributarios e incentivos para los inversores; entre otros (Proexport, 2012).

Con el potencial que tiene Colombia para aumentar las hectáreas de plantaciones comerciales se podrían sustituir las importaciones de madera y sus derivados, y además ganar participación en el mercado internacional. Las proyecciones de los diferentes mercados forestales parecen indicar que Colombia tiene muchas oportunidades, pues la demanda y el precio de la madera para diferentes usos seguirán en aumento. Para aprovechar estas oportunidades, es necesario que el Estado continúe con la definición de lineamientos para el desarrollo de las plantaciones forestales que permitan satisfacer las necesidades de materia prima para atender el mercado nacional e internacional. De la mano de las universidades y centros de investigación, el sector forestal debe ser más eficiente e innovar para ofrecer productos diferenciadores y con valor agregado. Pues darle valor agregado a las especies forestales que se cultivan comercialmente bajo principios de sostenibilidad en el país, desde conceptos de biorrefinería para bioenergía, biocombustibles y bioproductos, impactará de manera positiva la balanza económica y social, y promoverá nuevos desarrollos en materia industrial y de proceso en torno a la madera.

\section{CONCLUSIONES}

Colombia es uno de los países con mayor cobertura forestal y desarrollo forestal-industrial de América Latina; tiene un amplio potencial para aumentar las hectáreas de plantaciones comerciales que ayudaría a sustituir las importaciones de madera y sus derivados, y además ganar participación en el mercado internacional. Sin embargo, atendiendo conceptos de competitividad y globalización, es necesario buscar usos alternativos y sostenibles de las maderas comerciales como la bioenergía y los bioproductos, que generen mayor valor agregado a los recursos forestales. Al ser un tema poco examinado en el país, se crea la necesidad de estudiar la biorrefinería forestal como alternativa energética y fuente de productos de alto valor agregado para el sector. Por lo tanto, en este trabajo se revisaron algunos procesos específicos que pueden evaluarse como posibles estrategias de integración de la madera plantada en Colombia bajo el concepto de biorrefinería: procesos de gasificación de baja-media potencia, gasificación a escala industrial para producción de biocombustibles de alta calidad, procesos de mejoramiento de la madera como biocombustible sólido y la producción de biocarbón mediante métodos alternativos.

En cuanto a procesos de gasificación de biomasa con fines energéticos, se encuentra que, debido a la gran disponibilidad de recursos hídricos en el país, solo se considera el potencial de la biomasa como fuente no convencional de energía en zonas no interconectadas (ZNI). La baja demanda energética de esta población hace que la mejor opción en cuanto a tecnologías que operan con biomasa sea la gasificación en lecho fijo, que trae consigo grandes ventajas, pues al usar la biomasa en el mismo lugar en que se genera, disminuye los costos asociados a su almacenamiento y transporte, lo que hace que los costos de generación estén por debajo de los obtenidos con plantas diésel, que son las tecnologías utilizadas convencionalmente para generación en ZNI. 
La gasificación a escala industrial para producción de biocombustibles de alta calidad, como gas natural sintético (GNS) a partir de biomasa, ha sido poco estudiada en nuestro país y sería muy relevante, ya que se prevé el agotamiento del gas natural en los próximos años debido a la creciente demanda. Por lo tanto, el proceso de gasificación de biomasa puede dar respuesta a uno de los principales retos que enfrenta el país en encontrar la forma de agregar valor a la madera comercial mediante criterios medio ambientales, de eficiencia y sostenibilidad.

Por su parte, el biocarbón obtenido como producto de valor agregado en una biorrefinería sería de gran aplicabilidad en Colombia, bien sea para uso como fertilizante, como secuestrador de carbono, para tratamiento de aguas residuales, entre otros.

En cuanto a los procesos de mejoramiento de la madera como biocombustible sólido, se encuentra que la torrefacción es un proceso eficaz para mejorar las propiedades de la biomasa y aumentar su densidad energética y, por tanto, lograr mayor eficiencia en la gasificación. Dadas las perspectivas de crecimiento del sector forestal comercial en Colombia, una de las posibles alternativas de mercado para la madera es su exportación como biocombustible sólido. Para ello es necesario asegurar ciertas especificaciones como contenido energético, humedad, reactividad bajo procesos termoquímicos, entre otros, algunas de las cuales pueden mejorarse a través de pretratamientos como la torrefacción.

$\mathrm{Al}$ analizar las posibilidades que tendría la integración de plantaciones forestales comerciales en conceptos de biorrefinería en Colombia se encuentra que proyectos de este tipo se justifican desde las perspectivas técnica, energética, económica, social y ambiental; adicionalmente, se enmarcan dentro de diversos planes y políticas de gobierno que consideran el aprovechamiento energético de la biomasa como alternativa recursiva para el potencial silvicultural del país. Además, estos proyectos contribuirían con generación de empleo que podría ser de gran relevancia en un eventual proceso de paz.
Un aspecto adicional que asegura la calidad y pertinencia de dicha integración en Colombia es el potencial silvicultural del país, ya que en algunas de las principales especies forestales comerciales se obtienen muy buenos rendimientos, con la ventaja adicional de que dichos cultivos dedicados a biorrefinerías no compiten con la agricultura y pueden crecer en suelos degradados.

Por tanto, la unión estratégica entre universidades, centros de investigación y el sector forestal debe buscar la eficiencia e innovar para ofrecer productos diferenciadores y con valor agregado, aprovechando la existencia de nichos de mercado prácticamente inexplorados en Colombia, como la bioenergía y los bioproductos. Resulta necesario avanzar en el conocimiento que se tiene de las características y posibles usos de algunas especies forestales que se cultivan en el país desde conceptos de biorrefinería para bioenergía, biocombustibles y bioproductos con una perspectiva de sostenibilidad.

\section{ABREVIATURAS}

$\begin{array}{ll}\text { GNS } & \text { gas natural sintético } \\ \text { PAH } & \text { hidrocarburos poliaromáticos } \\ \text { PC } & \text { poder calórico } \\ \text { RE } & \text { relación equivalente } \\ \text { RI } & \text { relación de impregnación } \\ \text { TGA } & \text { Análisis termogravimétrico } \\ \text { ZNI } & \text { zonas no interconectadas }\end{array}$

\section{AGRADECIMIENTOS}

Este trabajo ha sido desarrollado con el apoyo económico del proyecto "Estrategias de integración de la madera plantada en Colombia en conceptos de biorrefinería termoquímica: análisis termodinámico y caracterización de bioproductos", financiado en la Convocatoria Programática Área Ingeniería y Tecnología 2014 - 2015 de la Universidad de Antioquia. 


\section{REFERENCIAS BIBLIOGRÁFICAS}

Adinata, D., Mohd, W., \& Aroua, M. K. (2007). Preparation and characterization of activated carbon from palm shell by chemical activation with $\mathrm{K} 2 \mathrm{CO} 3$. Bioresource Technology, 96, 145-149.

Agrocadenas. (2005). La cadena forestal y madera en Colombia: Una mirada global de su estructura y dinámica 1991-2005. 44p. Disponible en: www. fidamerica.cl/admin/docdescargas/centrodoc/centrodoc_1140.pdf

Anderson, N., Jones, J., Page-Dumroese, D., McCollum, D., Baker, S., Loeffler, D., \& Chung, W. (2013). A comparison of producer gas, biochar, and activated carbon from two distributed scale thermochemical conversion systems used to process forest biomass. Energies, 6(1), 164-183.

Asadullah, M., Miyazawa, T., Ito, S.-i., Kunimori, K., Yamada, M., \& Tomishige, K. (2004). Gasification of different biomasses in a dual-bed gasifier system combined with novel catalysts with high energy efficiency. Applied Catalysis A: General, 267(1-2), 95-102.

Bludowsky, T., \& Agar, D. W. (2009). Thermally integrated bio-syngas-production for Biorefineries. Chemical Engineering Research and Design, 87, 1328-1339

Bridgeman, T. G., Jones, J. M., Williams, A., \& Waldron, D. J. (2010). An investigation of the grindability of two torrefied energy crops. Fuel, 89, 3911-3918.

Buongiorno, J., Raunikar, R., \& Zhu, S. (2011). Consequences of increasing bioenergy demand on wood and forests: An application of the Global Forest Products Model. Journal of Forest Economics, 17(2), 214-229.

Buragohain, B., Mahanta, P., \& Moholkar, V. S. (2010). Thermodynamic optimization of biomass gasification for decentralized power generation and Fischer-Tropsch synthesis. Energy, 35(6), 2557-2579.

Ciolkozs, D., \& Wallace, R. (2011). A review of Torrefaction for bioenergy feedstock production. Biofuels, Bioproducts and Biorefining, 5(3), 317-329.

Consejo Nacional de Política Económica y Social (CNPES). (2013). Distribución de recursos para el certificado de incentivo forestal con fines comerciales (CIF de reforestación)-Vigencia 2013. Consejo Nacional de Política Económica y Social. 23 p.

Consonni, S., Katofsky, R. E., \& Larson, E. D. (2009). A gasification-based biorefinery for the pulp and paper industry. Chemical Engineering Research and Design, 87, 1293-1317.

Chambost, V., \& Stuart, P. R. (2007). Selecting the most appropriate products for the forest biorefinery. Industrial Biotechnology, 3(2), 112-119.

Chaouch, M., Dumarçay, S., Pétrissans, A., Pétrissans, M., \& Gérardin, P. (2013). Effect of heat treatment intensity on some conferred properties of different European softwood and hardwood species. Wood Science and Technology, 47(4), 663-673.

Chen, W.-H., Hsu, H.-C., Lu, K.-M., Lee, W.-J., \& Lin, T.-C. (2011). Thermal pretreatment of wood (Lauan) block by torrefaction and its influence on the properties of the biomass. Energy, 36(5), 3012-3021.

Chen, W.-H., \& Kuo, P.-C. (2010). A study on torrefaction of various biomass materials and its impact on lignocellulosic structure simulated by a thermogravimetry. Energy, 35(6), 2580-2586.

Chen, W.-H., \& Kuo, P.-C. (2011). Isothermal torrefaction kinetics of hemicellulose, cellulose, lignin and xylan using thermogravimetric analysis. Energy, 36(11), 6451-6460.

Cherubini, F. (2010). The biorefinery concept: Using biomass instead of oil for producing energy and chemicals. Energy Conversion and Management, 51, 1451-1421.

Dávila, J. A. (2013). Biorrefinerías en Colombia. Puertas abiertas, UN Radio, http://www.unradio.unal. edu.co/: Universidad Nacional de Colombia, Sede Manizales.

Demirbas, A. (2009). Biorefineries: Current activities and future developments. Energy Conversion and Management, 50, 2781-2801.

Demirbas, M. F., Balat, M., \& Balat, H. (2009). Potential contribution of biomass to the sustainable energy development. Energy Conversion and Management, 50(7), 1746-1760. 
Di Blasi, C. (2000). Dynamic behaviour of stratified downdraft gasfiers. Chemical engineering science, 55(15), 2931-2944.

Departamento Nacional de Planeación (DNP). (2010). Plan Nacional de Desarrollo 2010-2014. Bogotá: Departamento Nacional de Planeación-Colombia. $538 \mathrm{p}$.

Energy Information Administration (EIA). (2010). Key word energy statistics. Paris: U.S. Energy Information Administration. $82 \mathrm{p}$.

Energy Information Administration (EIA). (2011). International Energy Outlook 2011. Washington DC: U.S. Energy Information Administration. 659 p.

Esteves, B., Velez Marques, A., Domingos, I., \& Pereira, H. (2013). Chemical changes of heat treated pine and eucalypt wood monitored by FTIR. Maderas. Ciencia y Tecnología, 15, 245-258.

Esteves, B. M., Domingos, I. J., \& Pereira, H. M. (2011). Pine wood modification by heat treatment in air. BioResources, 3(1), 142-154.

Food and Agriculture Organization of the United Nations (FAO). (2006). Tendencias y perspectivas del sector forestal en América Latina y el Caribe. Roma: FAO. 200 p.

Food and Agriculture Organization of the United Nations (FAO). (2008). Contribution of the forestry sector to national economies 1990-2006. $180 \mathrm{p}$. Disponible en: www.fao.org/docrep/011/k4588e/ k4588e00.htm

Food and Agriculture Organization of the United Nations (FAO) (2012). El estado de los bosques del mundo 2012. 64 p. Disponible en http://www.fao. org/docrep/016/i3010s/i3010s.pdf

Fedemaderas. (2011). Acuerdo de competitividad cadena productiva forestal, madera, tableros, muebles y productos de madera. $26 \mathrm{p}$.

Fitz Patrick, M., Champagne, P., Cunningham, M. F., \& Whitney, R. A. (2010). A biorefinery processing perspective: Treatment of lignocellulosic materials for the production of value-added products. Bioresource Technology, 101(23), 8915-8922.

FPAC. (2011). The New Face of the Canadian Forest Industry. 12 p. Disponible en www.fpac.ca/ bio-pathways
Gasifipedia, N. (2013). Coal Power Gasification. Disponible en: http://www.netl.doe.gov/technologies/ coalpower/gasification/gasifipedia/

Ghalehno, M. D., \& Nazerian, M. (2011). Changes in the Physical and Mechanical Properties of Iranian Hornbeam Wood (carpinus betulus) with Heat Treatment. European Journal of Scientific Research, 51(4), 490-498.

Ghatak, H. G. (2011). Biorefineries from the perspective of sustainability: Feedstocks, products, and processes. Renewable and Sustainable Energy Reviews, 15, 4042-4052.

Guo, J., \& Lua, A. C. (1998). Characterization of chars pyrolyzed from oil palm stones for the preparation of activated carbons. Journal of Analytical and Applied Pyrolysis, 46, 113-125.

Hanaoka, T., Inoue, S., Uno, S., Ogi, T., \& Minowa, T. (2005). Effect of woody biomass components on air-steam gasification. Biomass and Bioenergy, 28(1), 69-76.

Hook, M., \& Aleklett, K. (2010). A review on coal-to-liquid fuels and its coal consumption. International Journal of Energy Research, 34. 848-864.

Ibrahim, R. H. H., Darvell, L. I., Jones, J. M., \& WiIliams, A. (2013). Physicochemical characterisation of torrefied biomass. Journal of Analytical and Applied Pyrolysis, 103, 21-30.

International Bank for Reconstruction and Development (IBRD). (1999). Energy from Biomass: A review of combustion and gasification technologies. Washington: The International Bank for Reconstruction and Development. $102 \mathrm{p}$.

IEA. (2003). Future Development of IGCC. Disponible en: http://www.iea-coal.org.uk/documents/82119/7089/ Future-developments-in-IGCC

Kumar, A., Jones, D. D., \& Hanna, M. A. (2009). Thermochemical Biomass gasification: A review of the current status of the technology. Energies, 2(3), 556-581

Kunze, C., \& Spliethoff, H. (2011). Modelling, comparison and operation experiences of entrained flow gasifier. Energy Conversion and Management, 52, 2135-2141. 
Kwapinski, W., Byrne, C. M. P., Kryachko, E., Wolfram, P., Adley, C., Leahy, J. J., ... Hayes, M. H. B. (2010). Biochar from Biomass and Waste. Waste and Biomass Valorization, 1(2), 177-189.

Lapuerta, M., Hernández, J. J., Pazo, A., \& López, J. (2008). Gasification and co-gasification of biomass wastes: Effect of the biomass origin and the gasifier operating conditions. Fuel Processing Technology, 89(9), 828-837.

Lenis, Y. A., Osorio, L. F., \& Pérez, J. F. (2013). Fixed bed gasification of wood species with potential as energy crops in Colombia: The Effect of the physicochemical properties. Energy Sources, Part A: Recovery, Utilization, and Environmental Effects, 35(17), 1608-1617.

Li, Z., Zhao, W., Zhang, F., \& Zhu, Q. (2008). Effect of corn stalk length on combustion characteristics in a fixed bed. Energy and Fuels, 22(3),2009-2014.

Linghong, Z., Xu, C. C., \& Champagne, P. (2010). Overview of recent advances in thermo-chemical conversion of biomass. Energy Conversion and Management, 51, 14, 969-982.

Lu, J., Yu, L., Zhang, X., Zhang, S., \& Dai, W. (2008). Hydrogen Production from a Fluidized-bed Coal Gasifier with In Situ Fixation of CO2 Part I: Numerical Model. Chemical Engineering \& Technology, 31(2), 197-207.

Márquez-Montesino, F., Zanzi-Vigouroux, R., Birbas, D., Aguiar-Trujillo, L., \& Ramos-Robaina, B. A. (2013). Carbón activado de semillas de tomate para adsorción de vapores de amoniaco, benceno y gasolina. Ingeniería Mecánica, 16(2), 83-90.

Maurstad, O., Herzog, H., Bolland, O., \& Beér, J. (2013). Impact of coal quality and gasifier technology on IGCC performance. Norwegian Research Council in the KLIMATEK program. Disponible en: http://sequestration.mit.edu/pdf/GHGT8_Maurstad.pdf

Melgar, A., Borge, D., \& Pérez, J. F. (2008). Estudio cinético del proceso de devolatilización de biomasa lignocelulósica mediante análisis termogravimétrico para tamaños de particula de 2 a $19 \mathrm{~mm}$. Dyna, 75, 123-131.
Ministerio de Ambiente, Vivienda y Desarrollo Territorial, Corporación Autónoma Regional de Risaralda, \& Unión-Europea. (2011). Pacto intersectorial por la madera legal en Colombia. $22 \mathrm{p}$.

Ministerio del Ambiente(Minambiente). (2000). Plan Nacional de Desarrollo Forestal. Ministerio del Medio Ambiente. 74 p.

Ministerio de Minas y Energía(Minminas). (2010). Programa de uso racional y eficiente de energía y fuentes no convencionales - PROURE. Bogotá: Ministerio de Minas y Energía. 151p.

Molina, S. (2012). Estudio de caracterización del residuo sólido carbonoso de gasificación de biomasa en el flujo arrastrado. Engineer, Universidad de Castilla-La Mancha. 86 p.

Nenoff, T. M., Berman, M. R., Glasgow, K. C., Cesa, M. C., \& Taft, H. (2012). Introduction to the special section on alternative energy systems: hydrogen, solar, and biofuels. Industrial \& Engineering Chemistry Research, 51(37), 11819-11820.

Octave, S., \& Thomas, D. (2009). Biorefinery: Toward an industrial metabolism. Biochimie, 91(6), 659-664.

Osorio, L. F., Del Valle, J. I., \& Restrepo, H. I. (2014). Valoración del potencial energético de núcleos forestales. En Universidad de Antioquia (Ed.), Biomasa forestal como alternativa energética: Análisis silvicultural, técnico y financiero de proyectos (1 ed., pp. 1-25). Medellín: Universidad de Antioquia.

Pérez, J. F., Lenis, Y., Rojas, S., \& Leon, C. (2012). Decentralized power generation through biomass gasification: a technical-economic analysis and implications by reduction of $\mathrm{CO}_{2}$ emissions. Revista de la Facultad de Ingeniería, 62, 157-169.

Phanphanich, M., \& Mani, S. (2011). Impact of torrefaction on the grindability and fuel characteristics of forest biomass. Bioresource Technology, 102(2), 1246-1253.

Prins, M. J., Ptasinski, K. J., \& Janssen, F. J. J. G. (2006). More efficient biomass gasification via torrefaction. Energy, 31(15), 3458-3470.

Proexport. (2012). Sector forestal en Colombia. Disponible en http://www.inviertaencolombia.com.co/ Adjuntos/Perfil_Forestal_2012.pdf 
Ptasinski, K. J., Prins, M. J., \& Pierik, A. (2007). Exergetic evaluation of biomass gasification. Energy, 32(4), 568-574.

Qian, K., Kumar, A., Patil, K., Bellmer, D., Wang, D., Yuan, W., \& Huhnke, R. L. (2013). Effects of biomass feedstocks and gasification conditions on the physiochemical properties of char. Energies, 6, 3972-3986.

Rincón-Narváez, P. C. (2010). The current state of nonconventional sources of energy and related perspectives. Ingeniería e investigación, 30, 165-173.

Robinson, P. J., \& Luyben, W. L. (2008). Simple dynamic gasifier model that runs in Aspen Dynamics. Industrial \& Engineering Chemistry Research, 47(20), 7784-7792

San Miguel, G., Domínguez, M. P., Hernández, M., \& Sanz-Pérez, F. (2012). Characterization and potential applications of solid particles produced at a biomass gasification plant. Biomass and Bioenergy, 47, 134-144.

Tay, D. H. S., Kheireddine, H., Ng, D. K. S., El-Halwagi, M. M., \& Tan, R. R. (2011). Conceptual synthesis of gasification-based biorefineries using thermodynamic equilibrium optimization models. Industrial \& Engineering Chemistry Research, 50(18), 10681-10695.

Taylor, G. (2008). Biofuels and the biorefinery concept. Energy Policy, 36(12), 4406-4409.

Unidad de Planeación Minero Energetica (UPME). (2007). Plan energético nacional. Contexto y estrategias. Bogotá: Ministerio de Minas y Energía. 240 p.

Unidad de Planeación Minero Energetica (UPME). (2009). Boletín Minero Energético №107. Bogotá: Ministerio de Minas y Energía. 77 p.

Unidad de Planeación Minero Energetica (UPME). (2010a). Formulación de un Plan de Desarrollo para las Fuentes no Convencionales de Energía en Colombia (PDFNCE). Bogotá: Ministerio de Minas y Energía. 173 p.
Unidad de Planeación Minero Energetica (UPME). (2010b). Plan de Abastecimiento para el Suministro y Transporte de Gas Natural. Disponible en http:// www1.upme.gov.co/

Unidad de Planeación Minero Energetica (UPME). (2010c). Plan nacional de desarrollo para las fuentes no convencionales de energía. Bogotá: Ministerio de Minas y Energía. 193 p.

Vaezi, M., Passandideh-Fard, M., Moghiman, M., \& Charmchi, M. (2012). On a methodology for selecting biomass materials for gasification purposes. Fuel Processing Technology, 98, 74-81.

Van der Stelt, M. J. C., Gerhauser, H., Kiel, J. H. A., \& Ptasinski, K. J. (2011). Biomass upgrading by torrefaction for the production of biofuels: A review. Biomass and Bioenergy, 35(9), 3748-3762.

Vishnu, M., \& Mala, R. (2012). Trends in bioconversion of lignocellulose: Biofuels, platform chemicals \& biorefinery concept. Progress in Energy and Combustion Science, 38(9), 522-550.

Vitasari, C., Jurascik, M., \& Ptasinski, K. (2011). Exergy analysis of biomass to synthetic natural gas (SNG) process via indirect gasification of various biomass feedstock. Energy, 36(6), 3825-3837.

Yu, G. W., Wang, Y. M., \& Xu, Y. Y. (2012). Modeling analysis of Shell, Texaco gasification technology's effects on water gas shift for Fischer-Tropsch process. Advanced Materials Research, 608, 1446-1453.

Zhang, Y., Li, B., Li, H., \& Liu, H. (2011). Thermodynamic evaluation of biomass gasification with air in autothermal gasifiers. Thermochimica Acta, 519(12), 65-71.

Zhao, Y., Wen, H., \& Xu, X. (2006). Conceptual design and simulation study of a co-gasification technology. Energy Conversion and Management, 47, 1416-1428

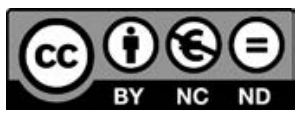

